\title{
EyePhy: Detecting Dependencies in Cyber-Physical System Apps due to Human-in-the-Loop
}

\author{
Sirajum Munir* \\ Bosch Research and Technology Center \\ Pittsburgh, PA 15203 \\ sirajum.munir@us.bosch.com
}

\author{
Mohsin Y. Ahmed, John A. Stankovic \\ University of Virginia \\ Charlottesville, VA 22904 \\ \{mohsin.ahmed, stankovic\}@cs.virginia.edu
}

\begin{abstract}
As app based paradigms are becoming popular, millions of apps are developed from many domains including energy, health, security, and entertainment. The US FDA expects that there will be 500 million smart phone users downloading healthcare related apps by 2015. Many of these apps are Cyber-Physical System (CPS) apps. In addition to sensing, communication, and computation, they perform interventions to control human physiological parameters, which can cause dependency problems as multiple interventions of multiple apps can increase or decrease each others effects, some of which can be harmful to the user. Such dependency problems occur mainly because each app is unaware about how other apps work and when an app performs an intervention to control its target parameters, it may affect other physiological parameters without even knowing it. We present EyePhy, a system that detects dependencies across interventions by having a closer eye on the physiological parameters of the human in the loop. To do that, EyePhy uses a physiological simulator HumMod that can model the complex interactions of the human physiology using over 7800 variables. EyePhy reduces app developers' efforts in specifying dependency metadata compared to state of the art solutions and offers personalized dependency analysis for the user. We demonstrate the magnitude of dependencies that arise during multiple interventions in a human body and the significant ability of detecting these dependencies using EyePhy.
\end{abstract}

\section{Categories and Subject Descriptors}

H.4 [Information Systems Applications]: Miscellaneous; C.3 [Special-Purpose and Application-Based Systems]: Real-time and Embedded Systems

\section{General Terms}

Algorithms

*This work was performed while the author was at the University of Virginia.

\section{Keywords}

Dependency analysis, Conflict detection, Human-in-the-loop, Interventions, Control

\section{INTRODUCTION}

As app based paradigms are becoming popular for smart phones and smart homes [23] [16], millions of apps are developed spanning many domains including energy, health, security, and entertainment. Currently, Apple and Google each have more than 1 million apps in their app stores. Among these apps, a significant number of medical apps already exist and newer apps are being developed every day. The US Food and Drug Administration expects that there will be 500 million smart phone users downloading healthcare related apps by 2015 [1]. Many of these app users are patients of one or more diseases. In 2005, $44 \%$ of all Americans had at least one chronic disease and $13 \%$ had three or more. By 2020, 157 million Americans are predicted to have more than one chronic disorder, with 81 million having multiple conditions, and many of them are likely to use healthcare apps [8]. Many of these apps perform interventions to control some physiological parameters of the human body.

This control part of medical apps poses several challenges because of two reasons. First, each app is developed independently and when it performs a control action, i.e., intervention on the human body, it does so without much knowledge about how the other apps work. Second, humans are an integral part of the feedback control loop. Therefore, when an app performs an intervention to control its target parameters, it may affect other physiological parameters without even knowing it since human body parts are very connected. As a result, if the user installs redundant apps, multiple apps may administer the same drug independently that may result in a drug overdose. Also, multiple interventions can increase or decrease each other's effects, some of which can be harmful to the user. To the best of our knowledge, no existing app engines available in the market perform any analysis across apps' control actions on the human body.

State of the art techniques, e.g., DepSys [23], require app developers to specify the effect of each actuation in order to detect the dependency at the actuator level. For example, an app that controls a humidifier specifies its effect $<h u$ midity $><$ increase $>$ while another app that controls a dehumidifier specifies its effect $<$ humidity $><$ decrease $>$ in the app metadata. Such a strategy works well in a home setting where there are only a few environmental parameters 
that can be affected by an intervention. App developers may even skip some effects in a home setting, e.g., an app that increases the room temperature by turning on an HVAC system may increase the humidity a little bit, but this effect is minor and is ignored and/or is not safety critical. However, we can not ignore such secondary effects in the context of a human body. So the state or art strategy of specifying the effect of actuations or intervention does not work because a human body has many interconnected parts. In other words, using current solutions app developers would have to specify hundreds or even thousands of parameters in order to specify the effects of an intervention on the human body.

We design EyePhy, a system that detects dependencies across interventions of human-in-the-loop CPS medical apps. We call it EyePhy as it has a closer eye on the physiological parameters of the involved human being. It uses a physiological simulator called HumMod [19] that can model the complex interactions of the human physiology using over 7800 variables capturing cardiovascular, respiratory, renal, neural, endocrine, skeletal muscle, and metabolic physiology. HumMod is constructed by the medical community from the empirical data collected from peer-reviewed physiological literature over more than 20 years, and it has been validated [19] on the criteria established in [26]. HumMod also permits specification of personalized information such as age, sex, weight, and medical condition, thus it is possible to model the physiology of people of both gender having various physical characteristics. Using a physiological simulator to detect dependencies has many advantages. First, it allows us to perform dependency analysis across a wide range of physiological parameters, e.g., HumMod's 7800 variables. Second, it enables us to take into account drug dosage and the time gap between the interventions in the dependency analysis. Some websites [2] [4] can be used to check for potential drug interactions, but they do not take into account these other issues. Also, they can't provide interaction analysis between a drug and a non-drug intervention, e.g., exercise, that our solution offers. Third, our dependency analysis is personalized, e.g., if someone has heart related problems, he can focus the dependency analysis on the heart. And fourth, all of these can be done without much effort from the app developers in specifying dependency metadata.

This work has five major research contributions. First, to the best of our knowledge, EyePhy is the first system to provide a comprehensive dependency analysis across human-inthe-loop medical apps by modeling the environment, i.e., the human body using over 7800 variables. Second, our solution takes into account drug dosage, time effect of interventions, and the time gaps between the dug dosages and interventions in the dependency analysis. Third, it reduces app developers' efforts in specifying dependency metadata compared to the state of art systems such as DepSys [23]. Fourth, it offers personalized dependency analysis for the user. Fifth, by using the HumMod simulator, we demonstrate the magnitude of dependencies that arise during multiple interventions in a human body and the significant ability of detecting these dependencies using EyePhy.

\section{RELATED WORK}

There are app based paradigms for smart phones (Android, iOS) and for smart homes, e.g., HomeOS [16] and DepSys
[23]. There are techniques that have been proposed to detect and resolve conflicts that arise when multiple apps try to control a sensor or an actuator. HomeOS can detect actuator level conflicts when multiple apps try to control the same actuator in a conflicting way, e.g., one app wants to turn on a light while another app wants to turn in off, and it resolves the conflict in favor of the higher priority app. DepSys can detect conflicts that occur across devices, e.g., one app is running a humidifier while another app is running a dehumidifier in the same room using additional meta data called effect, emphasis, and condition. It can resolve conflicts by understanding the contexts using the meta data. Also, it can automatically resolve control conflicts of the sensors by considering app priority, sensor resource availability constraint, and app requirements. These systems do not deal effectively with large numbers of secondary dependencies such as is found for human health.

PhysicalNet [27] provides a generic paradigm for managing and programming world-wide distributed heterogeneous sensor and actuator resources in a multi-user and multi-network environment. By allowing owners to specify fine grained access control and conflict resolution mechanism, PhysicalNet allows sharing of resources and increases the number of concurrent applications running on the devices. When multiple users simultaneously specify contradictory requirements on the same resource, PhysicalNet uses resolvers to resolve conflicts. Although some of the of conflicts that take place in a home occur due to human-in-the-loop, none of these techniques address the physiological aspect of the human being.

Human-in-the-loop is an active area of research. Humans are incorporated as a part of the feedback control loop in many systems including physiological control systems [11, 24], mobile sensing and computing systems [21, 25, 10, 28], thermal control systems [22], and robotic systems [20]. These works demonstrate that feedback control can be effectively used to control systems with direct feedback from the user. Some of the physiological control systems control the physiological parameters of the user. However, they do not consider the case of multiple interventions from multiple applications conflicting on the physiological parameters of the human.

There are many physiological models enabling the simulation of different functionalities of a human body including the pulmonary system [14] and cardiac metabolism [12]. However, these models are developed to understand the behavior of individual organs without providing the integration across different organ systems and simulation of the whole body. There are a few physiological simulators that integrate different organs. For example, Nottingham Physiology Simulator [18] allows simulation of cardiovascular, acid-base, respiratory, and renal physiological models. Guyton [17] and HUMAN [13] are two historical models of integrative physiology. QCP [9] is an extension of HUMAN and it incorporates cardiovascular, renal, respiratory, endocrine, and nervous systems. QCP is developed in $\mathrm{C}++$, which limits the ability to update the physiological models, e.g., changing or adding an equation requires recompilation of the whole simulator. HumMod [19] is developed to overcome this limitation as it uses XML files to describe the model parameters and the quantitative relationships among them. It uses over 7800 variables to capture cardiovascular, respiratory, renal, 


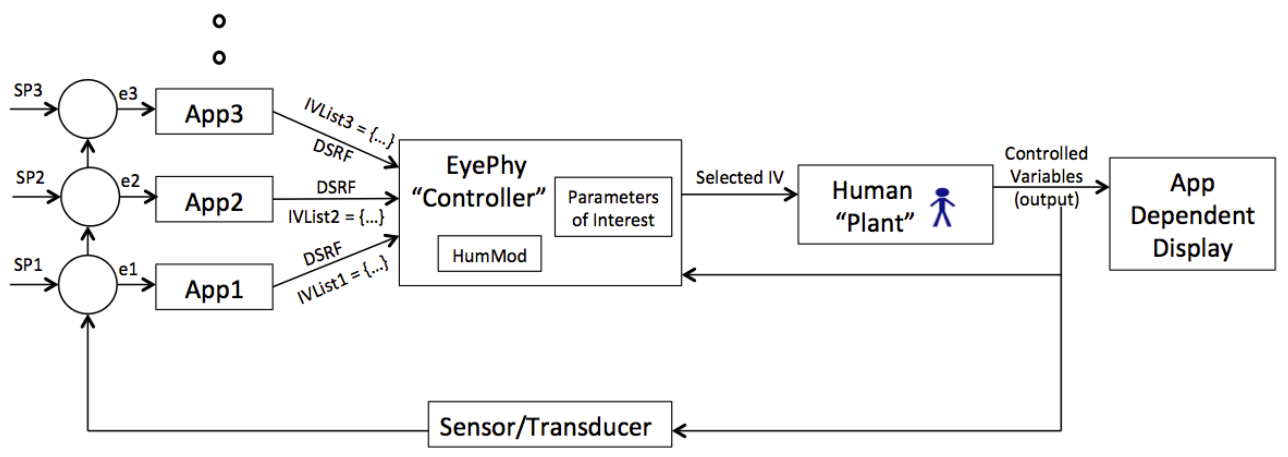

Figure 1: Runtime Dependency Detection by EyePhy

neural, endocrine, skeletal muscle, and metabolic physiology as a function of a person's age, weight, sex, and other personalized information. The model it uses is developed using the empirical data collected from peer-reviewed physiological literature. HumMod provides a model to understand the complex interactions of integrative human physiology and allows the simulation of different interventions on the human body. We choose to use this simulator as it is the leading physiological simulator in the medical community.

\section{APPROACH}

In this section, we describe our overall architecture, details on the classification of parameters and dependencies, the stakeholders and their responsibilities with suggested app metadata, and installation time and runtime dependency detection and resolution.

\subsection{Architecture}

We assume a centralized architecture for the app-based paradigm, which is common for smart phones and smart homes [16] [23]. Apps are run at the top layer by an app engine, which is the OS of a smart phone or a smart home. EyePhy runs as a service for the app engine, and detects dependencies at app installation time and at runtime.

At installation time a new app is compared to all previously installed apps by analyzing meta data and by running a simulation of the effects of possible interventions on the person for 3 hours (a tunable parameter) into the future. If there are serious conflicts the new app is not installed. More details are given in section 3.5.1.

The way EyePhy controls apps' interventions to a human at runtime is shown in Figure 1. Each app has one or more physiological parameters of interest that it monitors and tries to control. The app may have a setpoint that is used to compute an error, e.g., App1 has setpoint SP1 and it computes an error $e 1$ based on that. Then each app offers a list of suggested interventions to EyePhy, e.g., IVList1 is a list of one or more interventions suggested by App1. The list may contain the same drug with different dosage level, or different drugs, or other interventions. EyePhy uses HumMod to determine potential interactions with other previous interventions and selects an intervention that doesn't conflict with the previous ones. It projects the effects of all the interventions on the human for the next 3 hours (tunable). The selected intervention affects the physiological parameters, some of which are captured by the sensor/transducer and is used for determining the next interventions. An app uses its sensor data, if available, to update the values of physiological parameters of HumMod as shown DSRF (Dynamic Sensor Readings Feedback) in Figure 1. Figure 1 also shows how humans are part of the control loop. The controller also directly senses physiological responses of the user through available sensors to update the HumMod model, thus closing the loop. There are three novelties in our proposed system in Figure 1 compared to other human-in-the-loop feedback control systems. First, each app suggests a list of interventions instead of just one intervention, when possible. Second, EyePhy automatically detects interactions among the interventions not only at the current time, but out into a future time horizon and this is based on the patient current state. Third, it considers the parameters of interest of the user when detecting interactions, which allows dependency analysis to be personalized.

\subsection{Parameter and Dependency Classification}

HumMod models human physiology using over 7800 variables. If EyePhy uses all the variables for dependency detection, then almost any two interventions will interact with each other. We realize that all interactions are not necessarily harmful to the human body and hence to understand the dependencies, we categorize the parameters and dependencies as follows.

\subsubsection{High level and low level parameters}

High level parameters are the physiological parameters that are used to assess the general health of a person in most medical settings. These are also called vital signs. These parameters are as follows.

1. Body temperature

2. Heart rate

3. Blood pressure

4. Respiratory rate

5. Glucose

However, this list is extensible. We know the ranges (high, low) of each of these variables for a healthy body, which can be a function of age, sex, etc. Low level parameters are the other physiological parameters that are represented by over 7800 variables in HumMod. Unfortunately, the normal ranges of these parameter values of a healthy person are typically unknown. 


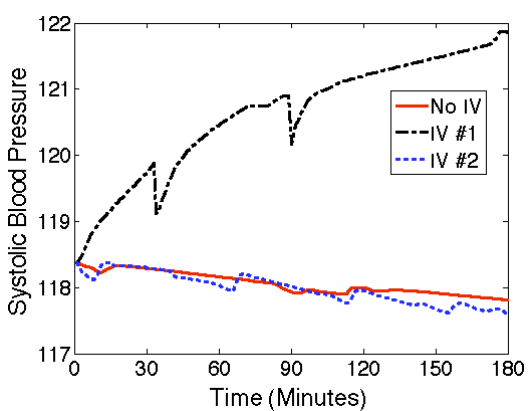

(a) Systolic Blood Pressure

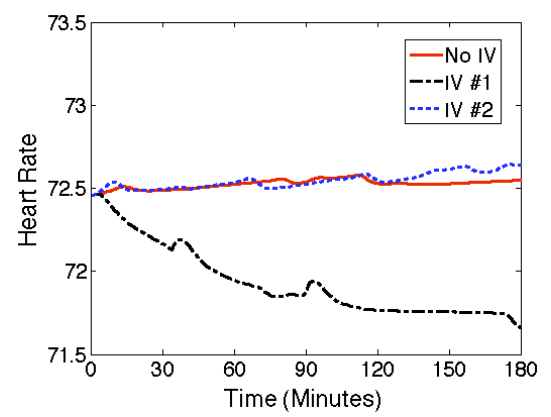

(b) Heart Rate

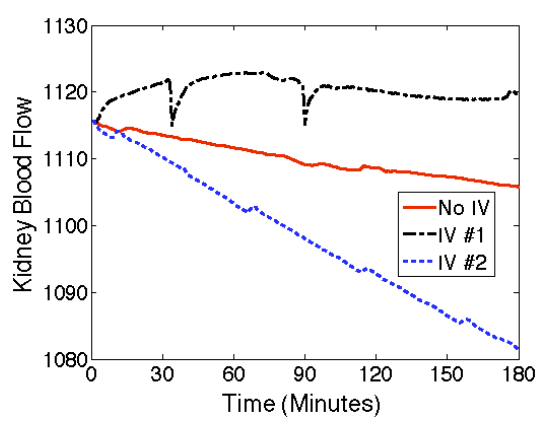

(c) Kidney Blood Flow

Figure 2: The effects of two interventions on the (a) Systolic Blood Pressure, (b) Heart Rate, and (c) Kidney Blood Flow.

Figure 2 shows how two different interventions can affect the high and low level physiological parameters. We consider two interventions, InterVention \#1 (IV \#1) and InterVention \#2 (IV \#2) in this analysis. We also consider the case without any intervention (shown as $N o I V$ in the figure). We use HumMod to simulate the effect of these interventions on the physiological parameters of a human body. The simulated person takes breakfast from 7 AM to $8 \mathrm{AM}$ in the morning. In case of $I V \# 1$, we administer a drug called Digoxin at 8 AM after eating the meal, which is used to treat heart failure, and simulate the physiological parameters for the next 3 hours. In case of $I V \# 2$, we administer a drug called Spironolactone, which is used to treat patients with hyperaldosteronism, at $8 \mathrm{AM}$ after eating the meal independently from the previous run and simulate the physiological parameters for the next 3 hours. In case of No $I V$, we just simulate the physiological parameters for 3 hours from $8 \mathrm{AM}$ without performing any intervention.

We show the effect of these interventions on three physiological parameters (systolic blood pressure, heart rate, and kidney blood flow) in Figure 2 for the next 3 hours from the $8 \mathrm{AM}$ of the simulated time. When we analyze the effect on the high level parameters (blood pressue and heart rate) we do not see any conflicts between the two interventions. Because, we see in the figure that $I V \# 1$ increases the blood pressure and decreases the heart rate, while $I V$ \#2 has minor effect on these parameters. Hence, they aren't considered conflicting. However, when we observe the effect of these two interventions on the kidney blood flow, which is a low level parameter, we see that they have completely opposite effect there. IV \#1 increases the kidney blood flow, while $I V$ \#2 decreases it. There are other high level parameters that we don't show in this figure due to lack of space. But it clearly shows that using only high level parameters is not sufficient to detect conflicting interventions.

\subsubsection{Numbers of Parameters}

We run an experiment to see the how many physiological parameters can be affected by an intervention and check whether it is feasible to ask the app developers to specify all the physiological parameters that can be affected by their interventions. We use interventions performed by App \#10, \#11, \#12, \#14, and \#18 from Table 1 in this study. HumMod does not provide any built in technique to determine whether a variable is affected by an intervention. Hence, we use a threshold $(\mathrm{X})$ for each parameter to determine that.

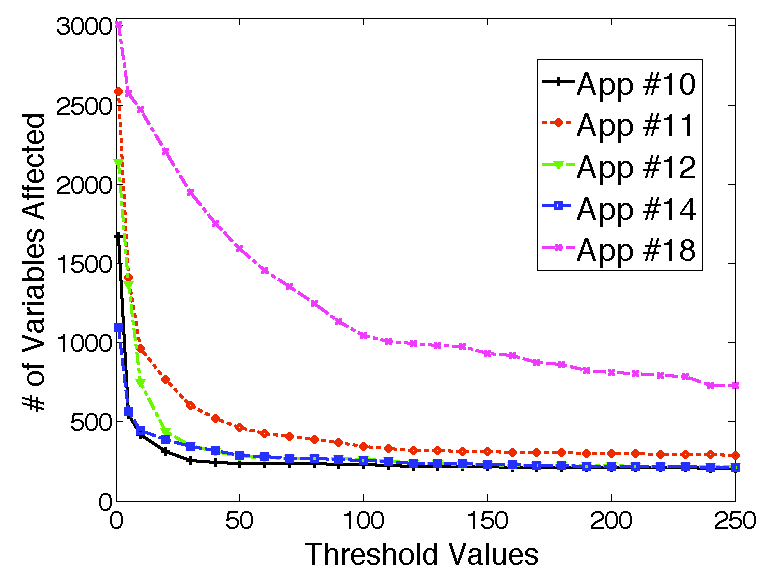

Figure 3: Number of variables affected by different apps' interventions with different threshold values

More specifically, for each intervention, we run the experiment two times, one with normal behavior (no intervention) and one with the intervention. We run both experiments for 3 hours of simulated time on the human body and collect all the variables' values once per minute. Assume that at minute $t 1$, for variable $v 1$, we observe $v 1=n 1$ for normal behavior and $v 1=i v 1$ for the intervention. Variable $v 1$ is considered affected by the intervention if $|n 1-i v 1| / n 1$ $>\mathrm{X}$ for at least one observation within the 3 hours. We count the number of variables affected by each intervention for different values of $\mathrm{X}$ ranging from $1 \%$ to $250 \%$ and show it in Figure 3. For the interventions that administer drugs (App \#10, \#11, \#12, \#14), we administer the app's first preferred dosage as mentioned in Table 1. For app \#18, we assume that the app suggests to do biking for 30 minutes.

We see in Figure 3 that when $\mathrm{X}$ is as low as 1\%, 1670, 2588, 2137, 1098, and 3010 variables are affected by App \#10, $\# 11$, \#12, \#14, and \#18, respectively. When $\mathrm{X}$ is $5 \%, 545$, $1412,1370,564$, and 2571 variables are affected by App \#10, $\# 11$, \#12, \#14, and \#18, respectively. When $\mathrm{X}$ is $250 \%$, $208,289,219,211$, and 727 variables are affected by App $\# 10, \# 11$, \#12, \#14, and \#18, respectively. This demonstrates that many physiological parameters are affected by each intervention even if we use high thresholds to choose the affected variables. It clearly shows that it is impractical to ask the app developers to specify all the physiological parameters that can possibly be affected by the app, and that we need an automated technique like EyePhy. 


\subsection{Stakeholders and Their Responsibilities}

To better describe EyePhy we list the various stakeholders and describe how they use the system.

- App developers: They develop apps and describe the primary and secondary dependencies using the high level parameters (just five parameters, unless extended) as app metadata. They can also specify low level parameters if known. In order to ensure all the app developers use the same terminology, we suggest using the parameter names specified in HumMod. They must also specify all the interventions performed by this app.

Dependency information involving a physiological parameter consists of two components: effect and dependency type. Effect of an intervention on a physiological parameter specifies the impact of the intervention on the parameter, which can be of three types as described below.

- increase: The parameter's value is increased by this intervention

- decrease: The parameter's value is decreased by this intervention

- null: The parameter's value is no affected by this intervention

Note that the effect can be both increase and decrease. The dependency type metadata can be either primary or secondary. We suggest the app developers to use the parameter names specified in the HumMod to describe the effects so that dependency metadata can be compared across apps. For example, if an app wants to perform an intervention that increases the heart rate, which causes a primary dependency, the app specifies the following dependency information in the app metadata.

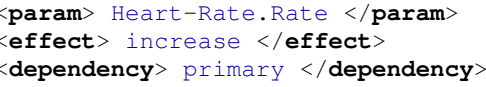

To specify interventions, app developers are suggested to use the parameter names specified in HumMod. For example, an app that wants to administer $10 \mathrm{mg}$ dosage of a drug called Midodrine specifies the intervention as shown below.

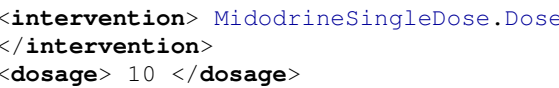

- Users: They install apps and the installed apps control their physiological parameters. If they have some basic knowledge of human physiology, they can choose parameters of their own interest, e.g., if someone has heart related problems, he can choose heart related low level parameters. However, we do realize that most users are not knowledgeable enough to choose parameters by their own.

- Caregivers and doctors: The caregivers and doctors can specify low level parameters of interest to focus the simulation. This allows personalized dependency analysis without requiring the patients to know about any low level parameters.

- Physiological model developers: They develop the physiological simulator HumMod that can simulate the effect of various interventions on the human body.
- EyePhy developers: We, the EyePhy developers, integrate the efforts of all other stakeholders to provide personalized dependency analysis for the users involving human-in-the-loop.

Note that Unspecified parameters are the parameters identified by EyePhy automatically because of the significant impact on these parameters by the apps' interventions. The unspecified parameters are presented to the users, caregivers, and doctors for additional consideration. If they select any of these parameters, the selected unspecified parameters are treated as caregiver specified parameters in the subsequent analysis.

\subsection{EyePhy Dependency Checking}

EyePhy considers all the specified parameters in the dependency analysis regardless of developer specified or caregiver specified. It also updates the list of unspecified parameters after performing the dependency analysis. EyePhy performs the following dependency checking at installation and runtime, respectively.

\subsubsection{Installation Time Checking}

When a new app is installed, EyePhy performs dependency analysis across all other installed apps for all the interventions specified in the new app. The new app is considered conflicting if at least one of the following two conditions is satisfied over a tunable future time horizon when comparing one of its intervention with another intervention from an already installed app.

(a) There is an opposite effect on at least one parameter, or

(b) There is a same effect on at least one parameter, where the joint intervention can exceed the normal range of the parameter

Two interventions are considered to have an opposite effect on a parameter when one intervention increases it while another intervention decreases it and a same effect on a parameter when they both increase or decrease the parameter value. Condition (a) is checked by analyzing the effect metadata, i.e., keywords increase/decrease for the developer specified parameters, and by simulating the intervention using HumMod for the caregiver specified parameters (both high level and low level). To determine whether an intervention increases or decreases a parameter using HumMod, EyePhy monitors the parameter values for the Window duration with and without the intervention. If the parameter value with the intervention reaches two standard deviations higher (lower) than the corresponding value without the intervention for at least one observation over the simulated time window, then EyePhy considers the effect is increase (decrease). The default value of the Window is 3 hours and can be changed if needed. Condition (b) is checked for all the high level parameters (regardless of specified or not) by simulating the joint intervention using HumMod and checking whether the parameter values stay within the acceptable range for the Window duration. However, EyePhy can't check for condition (b) for the low level parameters as the normal ranges of these parameters are not known. If it finds that the new app can conflict with a previously installed app due to an intervention, it alerts the user with the detailed information and suggests using caution to install and use the most recent app. Otherwise, it allows the new app to get installed without such a notification. 


\subsubsection{Runtime Checking}

At runtime, when an app requests to perform an actuation on the human body, that intervention request contains the suggested metadata related to the intervention. EyePhy checks whether the newly requested intervention can conflict with the previously granted interventions by checking for the conditions specified above in the installation time checking. However, there are two major differences from the installation time checking. First, EyePhy takes into account the time difference between the interventions at the runtime dependency analysis. To do that it uses the timestamps of interventions that are given input to EyePhy by the apps when they perform a direct actuation on the body or by the users when they listen to a suggestion, e.g., taking a drug or doing exercises. Second, at runtime, EyePhy maintains and updates the HumMod simulator based physiological model of the user by taking into account all the previously granted interventions and the current state of the patient based on sensor values. The apps that continuously monitor physiological parameters are allowed to provide input to EyePhy that is used to correct the physiological model. To determine whether the new intervention can conflict with the previously granted interventions, EyePhy checks whether the new intervention can cause any opposite effect with the most updated model (containing all the previous interventions) within the Window duration in at least one specified parameter using HumMod. It also checks whether the new intervention can cause any same effect with the most updated model and allowing the new intervention can exceed the normal range of at least one high level parameter, where the new intervention is started at the moment of request in the simulation.

An app's runtime intervention request contains a list of proposed interventions. EyePhy checks for conflicts for each intervention as mentioned above. It grants the first nonconflicting intervention in the list. If all the interventions in the list are conflicting, EyePhy doesn't grant the access of any intervention in the list. It notifies the user that the intervention request is denied and provides a brief explanation of why it is denied containing the parameter names where potential conflicts could occur. It also keeps a log of all the denied interventions to present to the caregivers and doctors. For each granted intervention, EyePhy updates a list of $K$ unspecified parameters that are most significantly affected (highest \% change from the without intervention value) by the intervention, which is later shown to the caregivers or doctors for additional consideration. Their chosen parameters are treated as caregiver specified parameters and used in subsequent dependency analysis.

\section{EVALUATION}

In this section, we demonstrate the conflict detection and resolution capability of EyePhy involving the human-in-theloop apps. We describe a list of human-in-the-loop apps for the evaluation, low level parameters of interest, experimental setup, and installation time and runtime dependency detection capability of EyePhy.

\subsection{App Selection}

In order to evaluate EyePhy, we need a list of human-inthe-loop apps. Although there are many healthcare related apps in Apple App Store and Google Play, the apps usually provide health related tips and do not perform any direct actuation on the human body due to strict FDA regulation. Hence, we design the 20 human-in-the-loop apps for the evaluation as shown in Table 1. These apps are representative of the research work in the wireless sensor networks area and the available healthcare apps in smart phones. When designing these apps, we consider many types of human-in-the-loop apps including the ones that just monitor health conditions with no interventions, apps that perform direct actuations on the human body, apps that provide health related suggestions, and apps that perform actuation on the environment that affects the human body. Among thousands of medical conditions, we only choose a few of the medical conditions (between App \#9 and App \#14), because HumMod can directly simulate their effects on the human body. Drug descriptions and dosages are obtained from [6]. We only use the 12 apps (from App\#9 to App\#20) from Table 1 in the subsequent evaluation since the interventions performed by these apps can be simulated by HumMod. Also, the medical conditions treated by these apps, e.g., blood pressure, heart rate, fluid control, glaucoma control, and insulin injection are very common as many elderly suffer from such simultaneous chronic conditions. We also determine the number of high level and low level parameters affected by these 12 apps by emulating their behaviors using HumMod for 3 hours and checking whether an intervention increases or decreases parameter values beyond two standard deviation of their normal values and show it in Table 1 . Other apps in Table 1 provides an overview about what else is proposed in the literature and available in the app markets. As HumMod supports more and more interventions, EyePhy will be able to perform dependency analysis across more apps.

\subsection{Low Level Parameters of Interest}

Based on the medical condition, specific low level physiological parameters need to be monitored for a user. In the evaluation, we consider three case studies focusing on three different parts of the human body.

1. 5 low level parameters related to the kidney: KidneyArcuateArtery.BloodFlow, Kidney-CO2.PCO2, Kidne y-EfferentArtery.VasaRectaOutflow, Kidney-Lactate. Flow, and Kidney-Fuel.TotalGlucoseUsed(Cals/Min).

2. 5 low level parameters related to the heart: LeftHea rt-BetaReceptors.Activity, LeftHeart-Flow.BloodFlo w, LeftHeart-Flow.O2Use, LeftHeart-Flow.PO2Effec t, and Heart-Ventricles.Rate.

3. 5 low level parameters related to the liver: Liver-O2 .BloodFlow, Liver-Fuel.GlucoseDelivered(Cals/Min), ADHClearance.Liver, GlycerolPool.LiverFARelease, and Liver-O2.InflowPO2.

\subsection{Experimental Setup}

EyePhy offers personalized dependency analysis and hence it is expected that the user will provide some basic information including his gender, age, weight, and height to personalize the HumMod physiological simulator. For the evaluation, we assume that the user is a 37 years male having 171 pounds of weight and $178 \mathrm{~cm}$ height, which is the default configuration of HumMod. We determine the normal ranges of the high level parameters for an average healthy person of this age from [7]. For example, for this age, the normal blood pressure is between $90 / 60 \mathrm{~mm} / \mathrm{Hg}$ and $120 / 80 \mathrm{~mm} / \mathrm{Hg}$, respiratory rate is between 12 and 18 breaths per minute, heart 


\begin{tabular}{|c|c|c|c|c|}
\hline ID\# & App Name & Description & \begin{tabular}{|c|c|c|} 
Parameter \\
High Level \\
\end{tabular} & $\begin{array}{l}\text { s Affected } \\
\text { Low Level } \\
\end{array}$ \\
\hline 1 & Lullaby & $\begin{array}{l}\text { This app uses sensors to keep track of sleep quality of the user. It uses sound, light, temperature, } \\
\text { and motion sensors to record the environmental condition during sleep. All these information is } \\
\text { presented to the user in a tablet that helps him to identify the potential causes of sleep disruption. }\end{array}$ & N/A & $\mathrm{N} / \mathrm{A}$ \\
\hline 2 & Fall Detector & $\begin{array}{l}\text { This app detects falls of the user using accelerometers and gyroscopes mounted on the body of the } \\
\text { user. The app notifies the caregivers when a fall is detected. }\end{array}$ & $\mathrm{N} / \mathrm{A}$ & $\mathrm{N} / \mathrm{A}$ \\
\hline 3 & Empath & $\begin{array}{l}\text { This app monitors the activity levels, sleep quality, speech prosody, and weight of the user to } \\
\text { detect depression. When a depression episode is detected, it turns on the lights and increases room } \\
\text { temperature by } 1 \text { degree } \mathrm{F} \text { of the occupied rooms. }\end{array}$ & $\mathrm{N} / \mathrm{A}$ & $\mathrm{N} / \mathrm{A}$ \\
\hline 4 & Kintense & $\begin{array}{l}\text { This app detects aggressive behavior of the user, e.g., hitting, kicking, pushing, and throwing using } \\
\text { a Kinect sensor. When such a behavior is detected, it warns the medical stuff. }\end{array}$ & $\mathrm{N} / \mathrm{A}$ & $\mathrm{N} / \mathrm{A}$ \\
\hline 5 & Musical Heart & $\begin{array}{l}\text { This app monitors the heart rate and activity level of the user, and recommends music to help the } \\
\text { user maintaining his target heart rate. }\end{array}$ & $\mathrm{N} / \mathrm{A}$ & $\mathrm{N} / \mathrm{A}$ \\
\hline 6 & Food Nutrition & $\begin{array}{l}\text { This app suggests eating nutritious foods. It also educates the user by providing nutrition facts of } \\
\text { over hundreds of foods containing all the vitamins and minerals information. }\end{array}$ & $\mathrm{N} / \mathrm{A}$ & $\mathrm{N} / \mathrm{A}$ \\
\hline 7 & Calorie Watcher & $\begin{array}{l}\text { This app allows the user to set a daily calorie budget and suggests food recipe to maintain the } \\
\text { budget. It also keeps a journal of all the food intakes. }\end{array}$ & $\mathrm{N} / \mathrm{A}$ & $\mathrm{N} / \mathrm{A}$ \\
\hline 8 & Pollen Alert & This app suggests to stay home during the period when the pollen level is high outside. & $\mathrm{N} / \mathrm{A}$ & $\mathrm{N} / \mathrm{A}$ \\
\hline 9 & $\begin{array}{l}\text { Blood Pressure } \\
\text { Control }\end{array}$ & $\begin{array}{l}\text { This app treats high blood pressure and fluid retention caused by various conditions, including } \\
\text { heart disease. It administers a drug called Chlorothiazide, which causes the kidneys to get rid of } \\
\text { unneeded water and salt from the body into the urine. The app administers this drug once a day } \\
\text { ( } 1000 \mathrm{mg} \text { dosage) in the morning or two times a day ( } 500 \mathrm{mg} \text { dosage) in the morning and in the } \\
\text { late afternoon with meals. }\end{array}$ & 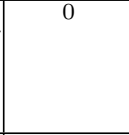 & 420 \\
\hline 10 & $\begin{array}{l}\text { Heart Rate Con- } \\
\text { trol }\end{array}$ & $\begin{array}{l}\text { This app helps control the heart rate. It administers a drug called Digoxin, which is used to } \\
\text { treat heart failure and abnormal heart rhythms. The app administers this drug once a day ( } 0.5 \\
\text { mg dosage) in the morning or two times a day ( } 0.25 \mathrm{mg} \text { dosage) in the morning and in the late } \\
\text { afternoon. }\end{array}$ & 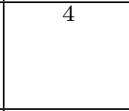 & 2901 \\
\hline 11 & Fluid Control & $\begin{array}{l}\text { The app is used to reduce the swelling and fluid retention caused by various conditions, including } \\
\text { heart or liver disease. It administers a drug called Furosemide, which causes the kidneys to get rid } \\
\text { of unneeded water and salt from the body into the urine. The app administers this drug once a } \\
\text { day ( } 80 \mathrm{mg} \text { dosage) in the morning or two times a day ( } 40 \mathrm{mg} \text { dosage) in the morning and in the } \\
\text { afternoon. }\end{array}$ & 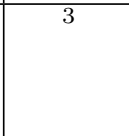 & 2085 \\
\hline 12 & $\begin{array}{l}\text { Low Blood Pres- } \\
\text { sure Control }\end{array}$ & $\begin{array}{l}\text { This app treats low blood pressure condition. It administers a drug called Midodrine, which } \\
\text { stimulates nerve endings in blood vessels, which tightens the blood vessels. As a result, blood } \\
\text { pressure is increased. It is also used to treat dizziness that occurs upon sitting up or standing. } \\
\text { The dosage is } 10 \mathrm{mg}, 3 \text { times a day with at least } 4 \text { hours interval in between. }\end{array}$ & 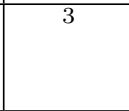 & 1532 \\
\hline 13 & $\begin{array}{ll}\text { Glaucoma Con- } \\
\text { trol }\end{array}$ & $\begin{array}{l}\text { This app treats glaucoma, a condition in which increased pressure in the eye can lead to gradual } \\
\text { loss of vision. The app administers Acetazolamide, which decreases the pressure in the eye. Aceta- } \\
\text { zolamide is also used to reduce the severity and duration of symptoms (upset stomach, headache, } \\
\text { shortness of breath, dizziness, drowsiness, and fatigue) of altitude (mountain) sickness. Acetazo- } \\
\text { lamide is used with other medicines to reduce edema (excess fluid retention) and to help control } \\
\text { seizures in certain types of epilepsy. Dosage is } 500 \mathrm{mg} \text { twice a day, one in the morning and one in } \\
\text { the afternoon. }\end{array}$ & 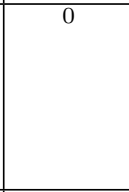 & 44 \\
\hline 14 & $\begin{array}{l}\text { Blood Pressure } \\
\text { Control II }\end{array}$ & $\begin{array}{l}\text { This app treats patients with hyperaldosteronism (a condition when the body produces too much } \\
\text { aldosterone, which is a naturally occurring hormone); low potassium levels; heart failure; and pa- } \\
\text { tients with edema (fluid retention) caused by various conditions, including liver, or kidney disease. } \\
\text { The app administers Spironolactone, which is used alone or with other medications to treat high } \\
\text { blood pressure. Spironolactone causes the kidneys to eliminate unneeded water and sodium from } \\
\text { the body into the urine, but reduces the loss of potassium from the body. The app administers } \\
\text { this drug once a day ( } 200 \mathrm{mg} \text { dosage) in the morning or two times a day (100 mg dosage) in the } \\
\text { morning and in the afternoon. }\end{array}$ & 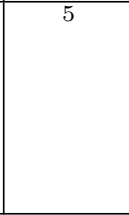 & 2877 \\
\hline 15 & Insulin Injection & $\begin{array}{l}\text { This app administers insulin based on the weight of the person and glucose in the blood level. } \\
\text { Assuming the weight of the person is } 171 \mathrm{lbs} \text {, it computes that the person needs } 42 \text { units of insulin } \\
\text { everyday. It is delivered as a mixture of short acting and long acting insulins in } 2 \text { injections } \\
\text { assuming the person has type- } 1 \text { diabetes. Two thirds of the daily dosage is given before the } \\
\text { breakfast and one third is given before the evening meal. }\end{array}$ & 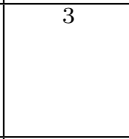 & 1461 \\
\hline 16 & $\begin{array}{l}\text { Activity } \text { Based } \\
\text { HVAC Control }\end{array}$ & $\begin{array}{l}\text { This app changes the room temperature based on the activities of daily living, e.g., while someone } \\
\text { is eating, the app reduces room temperature by } 1 \text { degree F. Changing the ambient temperature } \\
\text { may change the body temperature. }\end{array}$ & 0 & 82 \\
\hline 17 & \begin{tabular}{ll|}
$\begin{array}{l}\text { Humidity } \\
\text { trol }\end{array}$ & Con- \\
\end{tabular} & This app turns on the humidifier to increase the humidity if the humidity goes below a threshold. & 0 & 67 \\
\hline 18 & Physical Fitness & $\begin{array}{l}\text { This app suggests to go out for exercise in the afternoon. The suggestion could be to do biking or } \\
\text { to go to a gym to exercise on a treadmill. }\end{array}$ & 3 & 1713 \\
\hline 19 & $\begin{array}{ll}\text { Water } & \text { Intake } \\
\text { Monitor } & \end{array}$ & $\begin{array}{l}\text { This app monitors water intake in the body and suggests drinking more water if water intake is } \\
\text { lower than a threshold. }\end{array}$ & 0 & 159 \\
\hline 20 & $\begin{array}{l}\text { Nutrition Intake } \\
\text { Monitor }\end{array}$ & This app monitors nutrition intake in the body and suggests increasing nutrition intakes if needed. & 0 & 516 \\
\hline
\end{tabular}

\section{Table 1: A List of Human-in-the-Loop Apps}

rate is between 60 and 100 beats per minute, and temperature is between 97.8 and 99.1 degrees Fahrenheit.

\subsection{Static Analysis}

At the time of app installation, EyePhy performs installation time check to detect potential conflicts with the previously installed apps by analyzing the dependency information specified in the app metadata and running a simulation for 3 hours into the future. Figure 4 shows the probability of conflict between at least $\mathrm{j}$ apps when $\mathrm{i}$ apps are installed from the 12 apps (App \#9 to App \#20) in Table 1 using the high level parameters only. Figure 5 shows the case when we consider the low level parameters in addition to the high level parameters. For each value $\mathrm{x}$ in the $\mathrm{X}$ axis, we randomly select $\mathrm{x}$ apps 100 times and compute the probability of conflict between at least y apps, where $1 \leq \mathrm{y} \leq 5$ and $\mathrm{y}$ $\leq \mathrm{x}$, and show the average results in these figures. Figure 4 shows that when someone installs 2 apps, there is just $7 \%$ probability of conflict between them if we just use high level parameters for conflict detection. However, Figure 5 shows that this probability becomes $34 \%, 10 \%$, and $20 \%$ if we use low level parameters related to the kidney, heart, and liver, 


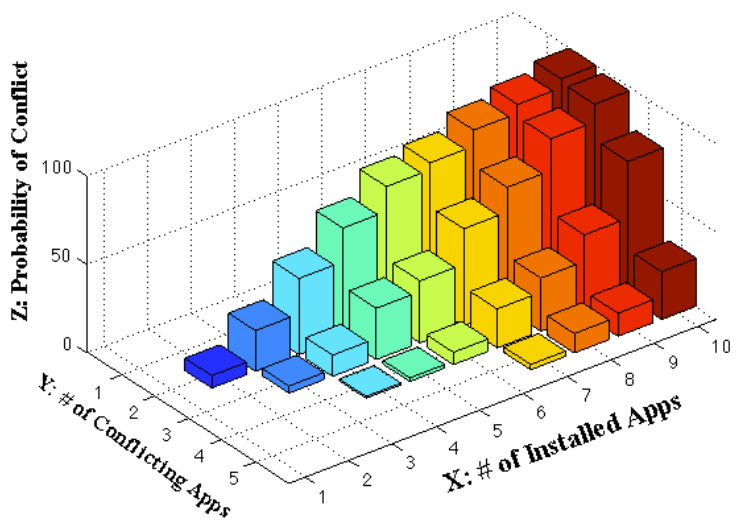

Figure 4: Installation time conflict detection on high level parameters

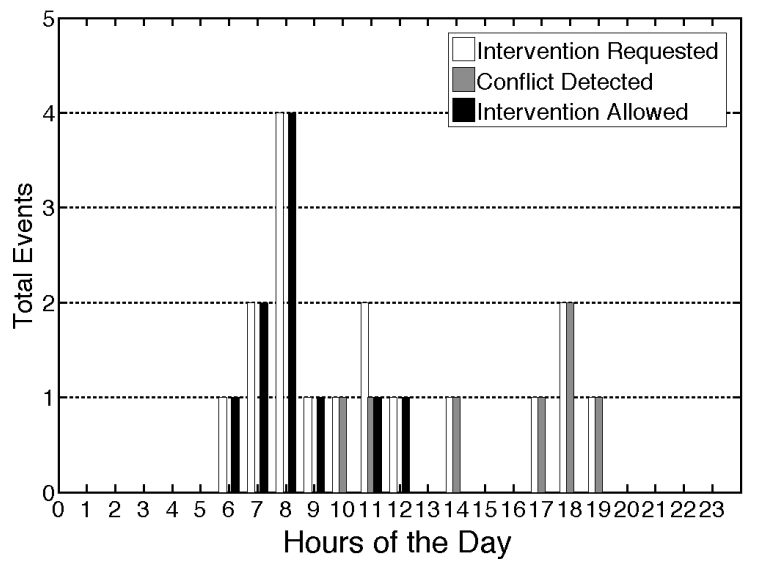

Figure 6: Runtime conflict detection on high level parameters

respectively in addition to the high level parameters. Hence, using low level parameters enables us to detect more potential conflicts in a personalized manner. We also see that installing more apps increases the probability of conflict. As we see in Figure 4, there is a $62 \%$ probability of conflict between at least 2 apps when someone installs 5 apps if we just use high level parameters. This probability becomes $98 \%, 75 \%$, and $88 \%$ if we also use low level parameters related to the kidney, heart, and liver, respectively as shown in Figure 5. When someone installs 10 apps, the probability of conflict between at least two apps is $99 \%$ when we only consider the high level parameters. If we also consider any of the three low level parameters, the probability becomes $100 \%$ for each of the three cases. These results show the severity of conflicts involving high level and low level physiological parameters among the human-in-the-loop apps and demonstrate the need for a runtime system for detecting these conflicts.

\subsection{Runtime Analysis}

In order to evaluate the runtime performance of EyePhy, we need to install some human-in-the-loop apps and let them perform interventions on one or more human subjects with and without EyePhy. However, doing simultaneous interventions from Table 1 on a human body may not be safe for the subject. Hence, instead of using real human subjects, we use HumMod to simulate the effect of interventions on the human body for the runtime analysis.
We assume that the user installs App \#9 to App \#20 in Table 1 in this evaluation. It may be questioned whether someone will install so many health related apps. We argue that more than 100,000 mobile medical apps are currently available [5], and in the Android app store itself, there are over 80 diabetes apps offering many functionalities including self monitoring of blood glucose recording, medication or insulin logs, and insulin dose calculator [15]. Although we do not have a statistic on the number of medical apps that people install in their smart phones, we see that average Android and iPhone users have 32 and 44 apps downloaded in their smart phones, respectively, based on a recent study [3]. Hence, it is not unreasonable to assume that some people will install a number of human-in-the-loop apps, often without a doctor's knowledge. We also expect this number to increase in the future.

The way these apps (App \#9 to App \#20) perform interventions is described in Table 1 . We run the experiment for a whole simulated day for an adult person using HumMod. The interventions depend on the lifestyle of the person. By default, in HumMod, the person sleeps from 10 PM to 6 AM and eats breakfast, lunch, and dinner between 7AM and 8 $\mathrm{AM}, 12 \mathrm{PM}$ and $1 \mathrm{PM}$, and $6 \mathrm{PM}$ and $7 \mathrm{PM}$, respectively. The apps that administer drugs take into account the meal times. App \#16 (Activity based HVAC control) also takes into account the times of eating the meals. As we use this setting, the apps perform interventions based on the time of the day by considering the lifestyle of the person. We compute the number of intervention requests, the number of conflicts detected, and the number of allowed interventions by EyePhy in every hour during the day. We show the results in Figure 6 when considering the high level parameters and in Figure 7 when considering the low level parameters related to the kidney, heart, and liver in addition to the high level parameters. For brevity, we call these four cases as high-level, kidney, heart, and liver cases, respectively in the following description. The interventions take place in the following order in the four cases.

- At 6 AM, App \#17 requests to perform an intervention to increase the room humidity, which is granted.

- At 7 AM, before the breakfast, App \#15 requests to inject insulin. Also, at 7 AM App \#16 requests to reduce room temperature by 1 degree $\mathrm{F}$ for the duration while the resident is having the breakfast. Both requests are granted in all the cases except the kidney case where we observe a conflict at the parameter Kidney-CO2.PCO2.

- At 8 AM, App \#9, \#10, \#13, and \#14 request to administer drugs. App \#9, \#10, and \#14's intervention request contains a list of two dosages, one with a daily dosage and the other one with a half daily dosage. If the daily dosage amount is granted, the app does not perform any other intervention for the rest of the day. However, if the half dosage is granted, it performs another intervention in the afternoon (6 PM for App\#9 and \#10, and 7 PM for App \#14) with the half daily dosage. If none of the dosages are granted at $8 \mathrm{AM}$, the apps retry the interventions at $9 \mathrm{AM}$, which may lead to administering a full daily dosage at $9 \mathrm{AM}$ or two half daily dosages at $9 \mathrm{AM}$ and $6 \mathrm{PM} / 7 \mathrm{PM}$. App \#13 has just one dosage in the request that it tries to 


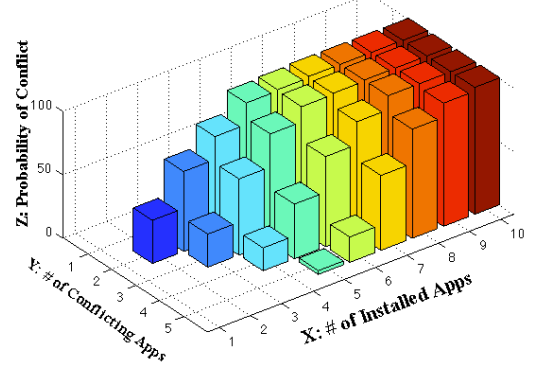

(a) Kidney

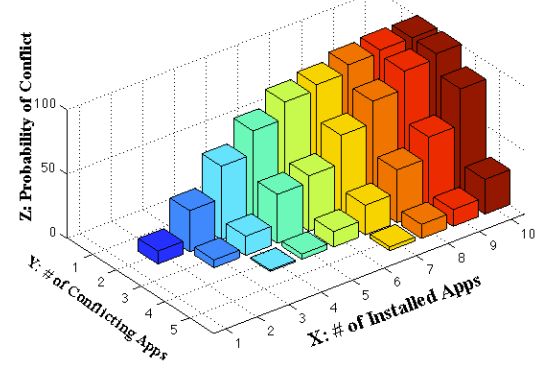

(b) Heart

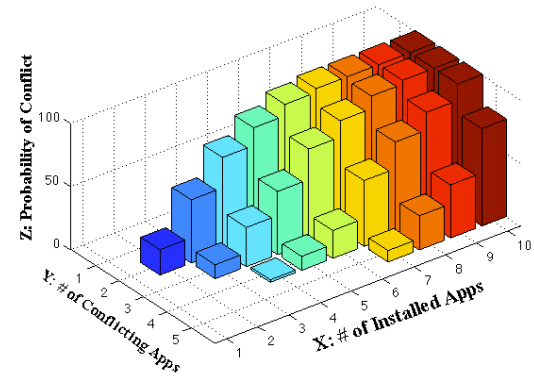

(c) Liver

Figure 5: Installation time conflict detection on low level parameters related to the (a) Kidney, (b) Heart, and (c) Liver in addition to the high level parameters.

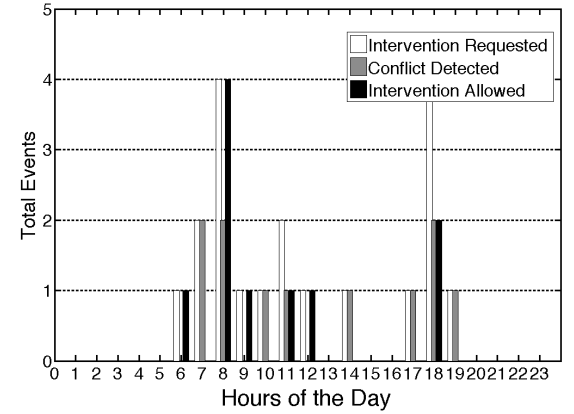

(a) Kidney

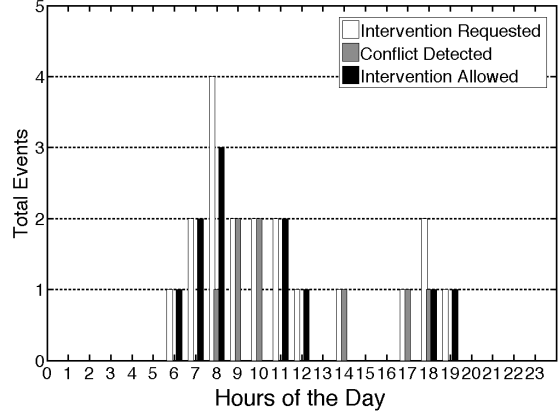

(b) Heart

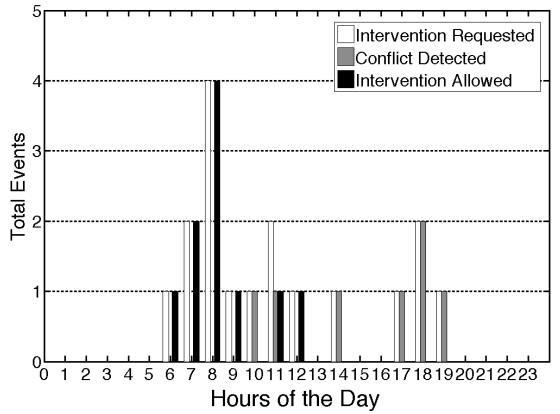

(c) Liver

Figure 7: Runtime conflict detection on low level parameters related to the (a) Kidney, (b) Heart, and (c) Liver in addition to the high level parameters.

administer at $8 \mathrm{AM}$ and at 7 PM. App \#13 and \#14's requests are granted in all the cases. App \#9, \#10's requests are granted in the high level case and the liver case. In case of the heart, App \#9's request is granted, but App \#10's request is denied because of a conflict at a heart related parameter LeftHeart-Flow.PO2Effect. In the case of kidney, App \#9 and \#10 both experience conflict for the daily dosage. However, both requests are resolved by using their half daily dosages that do not cause any conflict. The half daily dosage requests are granted.

- At 9 AM, App \#11 requests for interventions, where the intervention list contains a daily dosage and a half daily dosage. If none of the dosages are allowed, App \#11 retries at 10 AM. App \#11's request is granted in all the cases except the case with the heart where we observe a conflict at the parameter LeftHeart-Flow.PO2Effect. In case of the heart, App \#10 retries at 9 AM as its request at $8 \mathrm{AM}$ is denied, which is denied again due to a conflict at a heart related parameter.

- At 10 AM, App \#12 requests to perform an intervention, which is denied in all the cases due to a conflict with a high level parameter. In the case of the heart, App \#11 retries at $10 \mathrm{AM}$, which is denied again due to conflict at a heart related parameter.

- At 11 AM, App \#19 and \#20 request to perform interventions. App \#20's request is granted in all the cases. App \#19's request is denied in all the cases except the case with the heart, where it is granted.

We skip the description of the other interventions due to the lack of space. But the results clearly show that EyePhy's conflict detection and resolution can be personalized

\begin{tabular}{|l|l|l|l|}
\hline Parameter Types & $\begin{array}{l}\text { Total } \\
\text { Re- } \\
\text { quested } \\
\text { IVs }\end{array}$ & $\begin{array}{l}\text { Total } \\
\text { Conflicts } \\
\text { Detected }\end{array}$ & $\begin{array}{l}\text { Total } \\
\text { Allowed } \\
\text { IVs }\end{array}$ \\
\hline \hline High Level Parameters & 17 & 7 & 10 \\
\hline $\begin{array}{l}\text { High level and low level } \\
\text { parameters related to } \\
\text { the kidney }\end{array}$ & 19 & 11 & 10 \\
\hline $\begin{array}{l}\text { High level and low level } \\
\text { parameters related to } \\
\text { the heart }\end{array}$ & 19 & 8 & 11 \\
\hline $\begin{array}{l}\text { High level and low level } \\
\text { parameters related to } \\
\text { the liver }\end{array}$ & 17 & 7 & 10 \\
\hline
\end{tabular}

Table 2: Summary of the interventions of 24 hours by focusing the analysis to specific low level parameters.

A summary of the total number of requested interventions, the total number of conflicts detected, and the total allowed interventions of the day are shown in Table 2. It shows that when considering the high level parameters, a total of 17 interventions are requested by the apps in a day, out of which 7 result in conflicts and the other 10 interventions are allowed. It also shows the results when specific low level parameters are considered in addition to the high level parameters. When low level parameters related to the kidney are monitored in addition to the high level parameters, 19 intervention requests are generated by the apps in a day. Among these requests, 11 requests are detected as conflicts and 2 of the 11 requests are resolved as described above. In addition to these 2 resolved conflicts, 8 other interventions are allowed. It shows EyePhy's significant ability of detecting conflicts among interventions of apps at runtime as none 
of the conflicts could be detected without EyePhy. Note that detecting a single conflict involving human-in-the-loop can be crucial for making a life saving decision in some contexts.

\section{DISCUSSION}

Since EyePhy uses HumMod to simulate the interventions, the simulation may incur non-negligible energy consumption if EyePhy runs on a smart phone. Therefore, we run EyePhy in a desktop as HumMod is not ported to any smartphone platform yet. On the desktop, it takes about 2 seconds of real time to perform 10 minutes of simulation. However, as part of a future development plan, we envision a mobilecloud platform for EyePhy which will be more realistic and user-friendly for end users. In total, the runtime dependency analysis does not incur tremendous computational effort since we only need to do the simulation when an app requests an intervention, which happens only about 20 times in a day in our evaluation.

We did not perform any direct comparison with DepSys in the evaluation section. However, as described in Section 1, DepSys's employed strategy works well when the number of parameters affected by each intervention is limited. EyePhy reduces app developers efforts significantly in specifying dependency metadata. As an example, for each of the last 12 apps in Table 1, EyePhy requires specifying only five parameters and intervention description, whereas app developers would have to specify hundreds or even thousands of parameters if DepSys's employed strategy were used. We see a similar trend for the six apps in Figure 3, where different thresholds are used to determine whether a variable is affected by an intervention. Also, personalized dependency analysis is not addressed in DepSys.

\section{CONCLUSION}

Independently developed human-in-the-loop CPS apps pose new challenges for controlling human physiological parameters. This is primarily due to complex dependency issues of the apps' interventions. As far as we know, EyePhy is the first system to directly address these issues. EyePhy uses a novel approach by employing a medically accepted physiological simulator that can model the complex interactions of the human physiology using over 7800 variables. Our solution takes into account a wide range of physiological parameters at the time of dependency analysis as well as performs personalized dependency analysis over a time horizon. The end result permits a significant number of conflicts among the interventions to be detected. This is a major step towards the complete analysis of human-in-the-loop apps.

\section{ACKNOWLEDGEMENT}

This work was supported, in part, by NSF grant CNS-1239483 and by Microsoft SEIF.

\section{REFERENCES}

[1] http://lifescientist.com.au/content/biotechnology/article/therise-of-smartphone-health-and-medical-apps-1072193834.

[2] http://reference.medscape.com/drug-interactionchecker.

[3] http://velositor.com/2012/02/27/the-average-iphone-user-has44-apps-on-their-device-versus-only-32-apps-for-androidsmartphone-users/.

[4] http://www.drugs.com/drug_interactions.php.

[5] http://www.informationweek.com/regulations/lawmakers-tryto-sharpen-fda-focus-on-healthcare-apps/d/d-id/1112095?
[6] http://www.nlm.nih.gov/medlineplus/druginformation.html.

[7] http://www.nlm.nih.gov/medlineplus/ency/article/002341.htm.

[8] Tackling the burden of chronic diseases in the usa. The Lancet 373(9659):185, Jan. 2009

[9] S. R. Abram, B. L. Hodnett, R. L. Summers, T. G. Coleman, and R. L. Hester. Quantitative circulatory physiology: an integrative mathematical model of human physiology for medical education. Advances in Physiology Education, 31(2):202-210, 2007.

[10] M. Y. Ahmed, S. Kenkeremath, and J. Stankovic. Socialsense: A collaborative mobile platform for speaker and mood identification. In EWSN, 2015.

[11] D. Arney, M. Pajic, J. M. Goldman, I. Lee, R. Mangharam, and O. Sokolsky. Toward patient safety in closed-loop medical device systems. In ICCPS, 2010.

[12] D. A. Beard. Modeling of oxygen transport and cellular energetics explains observations on in vivo cardiac energy metabolism. PLoS Comput Biol, 2(9):107, 2006.

[13] T. G. Coleman and J. E. Randall. Human-a comprehensive physiological model. Technical report, The Physiologist, 1983.

[14] A. Das, Z. Gao, P. P. Menon, J. G. Hardman, and D. G. Bates A systems engineering approach to validation of a pulmonary physiology simulator for clinical applications. Journal of The Royal Society Interface, 8(54):44-55, 2011.

[15] A. P. Demidowich, K. Lu, R. Tamler, and Z. Bloomgarden. An evaluation of diabetes self-management applications for android smartphones. volume 18, 2012.

[16] C. Dixon, R. Mahajan, S. Agarwal, A. Brush, B. Lee, S. Saroiu, and P. Bahl. An operating system for the home. In NSDI, 2012.

[17] A. C. Guyton, T. G. Coleman, and H. J. Granger. Circulation: Overall regulation. Annual Review of Physiology, 34(1):13-44, 1972

[18] J. G. Hardman, N. M. Bedforth, A. B. Ahmed, R. P. Mahajan, and A. R. Aitkenhead. A physiology simulator: validation of its respiratory components and its ability to predict the patient's response to changes in mechanical ventilation. British Journal of Anaesthesia, 81(3):327-32, 1998.

[19] R. Hester, A. Brown, L. Husband, R. Iliescu, W. A. Pruett, R. L. Summers, and T. Coleman. Hummod: A modeling environment for the simulation of integrative human physiology. Frontiers in Physiology, 2(12), 2011.

[20] D.-J. Kim and A. Behal. Human-in-the-loop control of an assistive robotic arm in unstructured environments for spinal cord injured users. In $H R I, 2009$.

[21] X. Liu, H. Ding, K. Lee, L. Sha, and M. Caccamo. Feedback fault tolerance of real-time embedded systems: issues and possible solutions. SIGBED Rev., April 2006.

[22] J. Lu, T. Sookoor, V. Srinivasan, G. Gao, B. Holben, J. Stankovic, E. Field, and K. Whitehouse. The smart thermostat: using occupancy sensors to save energy in homes. In SenSys, 2010.

[23] S. Munir and J. A. Stankovic. Depsys: Dependency aware integration of cyber-physical systems for smart homes. In ICCPS, 2014 .

[24] S. Munir, J. A. Stankovic, C.-J. M. Liang, and S. Lin. Cyber physical system challenges for human-in-the-loop control. In Feedback Computing, 2013.

[25] S. Munir, J. A. Stankovic, C.-J. M. Liang, and S. Lin. Reducing energy waste for computers by human-in-the-loop control. IEEE Transactions on Emerging Topics in Computing, to appear.

[26] R. L. Summers, K. R. Ward, T. Witten, V. A. Convertino, K. L. Ryan, T. G. Coleman, and R. L. Hester. Validation of a computational platform for the analysis of the physiologic mechanisms of a human experimental model of hemorrhage. Resuscitation, 80(12), 2009.

27] P. A. Vicaire, Z. Xie, E. Hoque, and J. A. Stankovic. Physicalnet: A generic framework for managing and programming across pervasive computing networks. In $R T A S$, 2010.

28] T. Yan, V. Kumar, and D. Ganesan. Crowdsearch: Exploiting crowds for accurate real-time image search on mobile phones. In MobiSys, 2010. 